\title{
Fabrication, caractérisation et modélisation de micropoutres multimorphes intégrant un film piézoélectrique d'AIN comme actionneur
}

\author{
Alexandru Andrei ${ }^{1}$, Katarzyna Krupa ${ }^{1}$, Michal Jozwik ${ }^{2}$, Patrick Delobelle ${ }^{3, a}$, \\ Laurent Hirsinger $^{3}$, Christophe Gorecki ${ }^{1}$, Lukasz Nieradko ${ }^{1}$ Et Cathy MeunieR $^{4}$ \\ 1 LOPMD, FEMTO-ST, UMR CNRS 6174, Université de Franche-Comté, 16 route de Gray, 25000 Besançon, France \\ 2 Institute of Micromechanics and Photonics, Université de Technologie de Varsovie, 8 sw. A. Bokoli, 02.525 Varsovie, Pologne \\ 3 LMARC, FEMTO-ST, UMR CNRS 6174, Université de Franche-Comté, 24 chemin de l'Épitaphe, 25000 Besançon, France \\ 4 CREST, FEMTO-ST, UMR CNRS 6174, Université de Franche-Comté, 4 place Tharradin, 25200 Montbéliard Cedex, France
}

Reçu le 15 mars 2007, accepté le 7 juin 2007

\begin{abstract}
Résumé - Cet article présente les potentialités de films d'AlN piézoélectriques comme éléments actionneurs pour les MEMS. Le cas de micropoutres constituées d'un empilement de différents films (électrode/AlN/électrode/substrat Si) est présenté. Un dispositif interférométrique de type Twyman-Green permet de quantifier précisément les déformées et les déplacements ce qui permet de calculer, à l'aide des équations non simplifiées, différentes grandeurs physiques des films d'AlN, par exemple le module d'Young lié à l'orientation (002) des cristallites, les contraintes résiduelles consécutives au mode d'élaboration, le coefficient de dilatation $\alpha$ ainsi que le coefficient piézoélectrique $d_{31}$. On retrouve sensiblement les valeurs du matériau massif.
\end{abstract}

Mots clés : Nitrure d'aluminium / film mince / contrainte résiduelle / coefficient piézoélectrique

\begin{abstract}
Fabrication, characterization and modelling of multilayered cantilevers with piezoelectric AlN ceramic as actuator. This paper treats a wide range of subjects related to the use of AlN as actuation layer in MEMS, from its deposition conditions to accurate interferometric device characterization and physical parameters extraction. The case of AlN driven multilayered cantilevers has been considered. Parameters such as Young's modulus associated to the (002) orientation of the crystallites, residual thin film stresses, thermal expansion coefficient $\alpha$ and piezoelectric coefficient $d_{31}$ have been calculated using non approximated equations capable of taking into account multiple film stacking. The well oriented thin film exhibit approximately the same properties as the bulk material.
\end{abstract}

Key words: Aluminium nitride / thin film / residual stress / piezoelectric coefficient

\section{Introduction}

Le nitrure d'aluminium (AlN) est un semi conducteur du type III-V à large bande gap directe et qui est utilisé dans de nombreux domaines d'applications. Ses propriétés piézoélectriques associées à la vitesse élevée de propagation des ondes ultrasonores, sa bonne stabilité thermique et sa faible température de déposition (en dessous de $400^{\circ} \mathrm{C}$ ) compatible avec le post-traitement de la technologie des circuits imprimés, en font un bon candidat pour les applications ondes acoustiques de surface dans la bande

\footnotetext{
a Auteur correspondant :

patrick.delobelle@univ-fcomte.fr
}

du GHz (SAW) [1,2] et des filtres d'onde acoustique volumique (BAW) [3,4]. En marge du marché toujours croissant de la communication sans fils, l'AlN a aussi été utilisé comme capteur de pression, de gravimétrie et même comme capteur chimique en utilisant la propagation des ondes de Lamb sur des membranes AlN/Si $[5,6]$. Pour ce qui concerne l'actionnement, les films de PZT élaborés avec la technique sol-gel sont préférentiellement utilisés pour leur fort coefficient piézoélectrique (9 à 10 fois supérieur à celui de l'AlN) et leur possibilité d'être déposé en couche épaisse. Cependant, l'AlN reste une bonne alternative pour l'actionnement de certains MEMS [7] en offrant un certain nombre d'avantages [8]. En plus de sa compatibilité avec la technologie des circuits intégrés liée 
à sa faible température de déposition, l'AlN possède une excellente stabilité chimique, une constante diélectrique cent fois plus faible que celle du PZT, un champ de claquage élevé $\left(>800 \mathrm{KV} . \mathrm{cm}^{-1}\right)$ et il ne requiert aucune polarisation préalable puisqu'il n'est pas ferroélectrique. Ainsi l'AlN semble prometteur pour les applications où de faibles pertes diélectriques, une faible dérive thermique et un grand rapport signal sur bruit sont requis. L'objectif du présent travail a été de développer des micropoutres de haute qualité à base d'AlN pouvant être utilisées dans des MEMS plus complexes, comme la microscopie optique champ proche sur puce proposée par Bargiel et al. [9]. En plus du procédé d'élaboration, cette étude se focalise sur le contrôle de l'orientation des cristallites des films déposés ainsi que sur le niveau des contraintes résiduelles. En général, une orientation (002) des cristallites est facilement obtenue par déposition en phase vapeur (PVD) comme la pulvérisation cathodique. Cependant, l'orientation (002) conduit à des propriétés électromécaniques légèrement plus faibles que pour l'orientation (100) [10]. Il y a de très nombreux paramètres qui contrôlent la croissance du film [10-16], comme la distance entre la cible d'aluminium et le substrat, la pression de gaz, la puissance du réacteur,... Dans cette étude on s'intéresse à l'influence sur la cristallographie de la nature du métal de l'électrode inférieure ( $\mathrm{CrNi}, \mathrm{Pt}$ ) sur laquelle est déposé le film, le niveau de contrainte et les propriétés piézoélectriques des films synthétisés.

\section{Procédures expérimentales}

\subsection{Fabrication des structures}

Les microsystèmes poutres sont fabriqués à partir de wafers de Si de $3^{\prime \prime}$ orientés (100) et de $380 \mu \mathrm{m}$ d'épais. Les étapes de fabrication sont présentées sur la figure 1a. Après avoir réalisé une membrane de Si de $15 \mu \mathrm{m}$ d'épais par attaque $\mathrm{KOH}$ sur la face arrière du wafer (étape 1), sur sa face supérieure trois films sont successivement déposés, deux épaisseurs en CrNi par PVD $(0,15 \mu \mathrm{m}$ à l'étape 2 et $0,35 \mu \mathrm{m}$ pour l'étape 3$)$ entre lesquelles se situe le film d'AlN. L'alliage $\mathrm{Cr}_{50 \%} \mathrm{Ni}_{50 \%}$ a été pulvérisé sur un substrat chaud $\left(200{ }^{\circ} \mathrm{C}\right)$ pour une puissance de $230 \mathrm{~W}(0,5 \mathrm{~A})$ sous une pression d'Ar de 0,93 Pa. Le film d'AlN a été déposé (étape 3) dans un réacteur DC de pulvérisation réactive pulsée sur un substrat non chauffé placé à $70 \mathrm{~mm}$ de la cible en $\mathrm{Al}$ avec une puissance de $650 \mathrm{~W}$ et un rapport gaz $\mathrm{Ar} / \mathrm{N}_{2}$ de $0,65 \mathrm{sccm} / 6 \mathrm{sscm}$ pour une pression de $0,65 \mathrm{~Pa}$. Ces conditions conduisent à une vitesse de déposition d'environ $17 \mathrm{~nm} \cdot \mathrm{min}^{-1}$, ce qui est en accord avec les résultats de Kusaba et al. [13]. Deux wafers différents ont été utilisés avec des épaisseurs d'AlN différentes, $1 \mu \mathrm{m}$ pour le wafer 1 et $1,4 \mu \mathrm{m}$ pour le wafer 2 .

La température du substrat étant seulement dépendante de l'échauffement du plasma, sa valeur n'excède jamais $130{ }^{\circ} \mathrm{C}$ à la fin du processus de déposition de l'AlN.

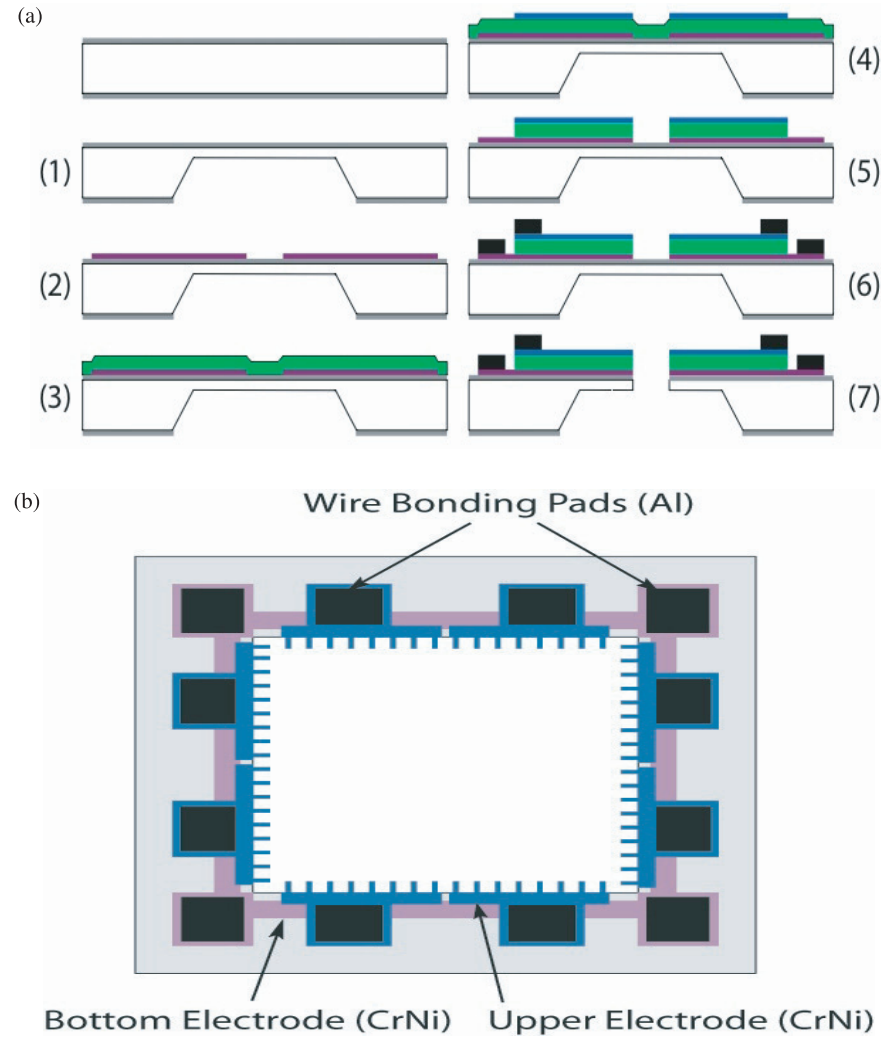

Fig. 1. Étapes de fabrication des microsystèmes poutres (a) et exemple de réalisation (b).

L'électrode supérieure en $\mathrm{CrNi}$, réalisée à l'étape 4, a été utilisée comme masque dur durant les attaques de l'AlN et du Si (étapes 5 et 7 ). Une étape intermédiaire (6) est nécessaire pour la déposition et la mise en forme de plots d'aluminium de $0,5 \mu \mathrm{m}$ d'épais pour le soudage des fils des connexions électriques. L'AlN a été attaqué en utilisant une solution liquide basique. Le Si qui n'est pas protégé par le masque en CrNi est attaqué par RIE, transformant la membrane initiale en un réseau de micropoutres encastrées-libres. Elles ont une largeur constante de $50 \mu \mathrm{m}$ et des longueurs variables de 200 à $900 \mu \mathrm{m}$. À noter que l'épaisseur exacte des poutres

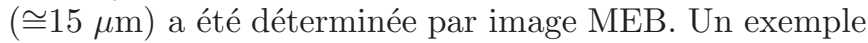
de réseau de poutres de $200 \mu \mathrm{m}$ de long est donné sur la figure 1b. L'actionnement des poutres est réalisé par l'application d'une tension $(\leqslant 15 \mathrm{~V})$ entre les électrodes supérieure et inférieure. La figure 2 présente une image MEB du microsystème une fois découpé et connecté à son environnement.

Précédant la fabrication de ces systèmes, deux séries de structures tests ont été réalisées. Elles permettent la mesure des contraintes dans les différents films suivant les étapes du procédé, ainsi que d'étudier l'influence de la nature de l'électrode inférieure sur l'amplitude de ces contraintes. Ces structures tests sont des réseaux de poutres de $13 \mu \mathrm{m}$ d'épais, de $0,5 \mathrm{~mm}$ de large pour des longueurs comprises entre 0,5 et $3,5 \mathrm{~mm}$. Deux types d'électrode inférieure ont été testés; $0,16 \mu \mathrm{m}$ d'épais de CrNi et 0,24 $\mu \mathrm{m}$ d'épais de Pt. 


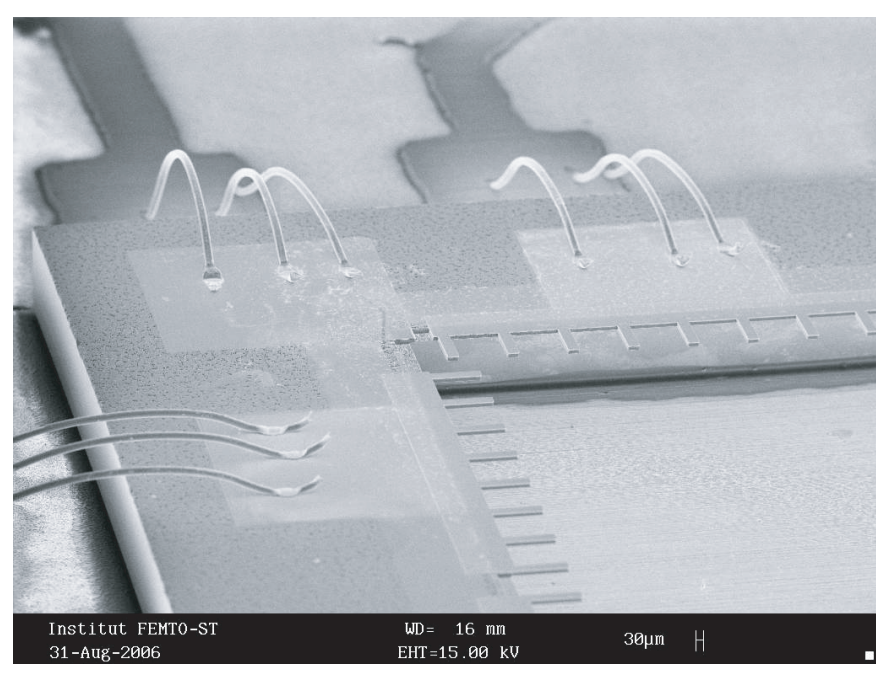

Fig. 2. Image MEB du système avec son environnement.

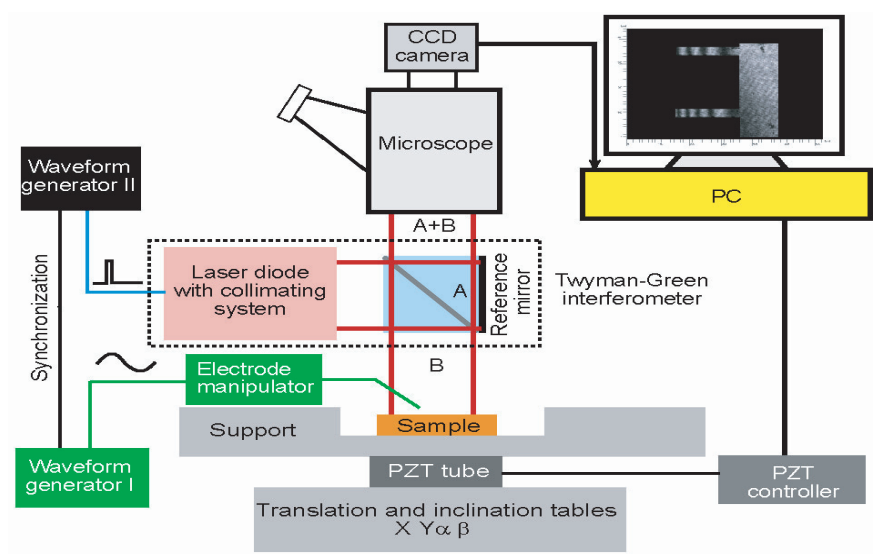

Fig. 3. Schéma de la plate forme interférométrique.

Le CrNi a été déposé de la même façon que pour les microsystèmes alors que le Pt a été pulvérisé sur un substrat non chauffé sous une puissance de 120 W (0,22 A) et une pression d'Ar de 1,1 Pa. Après la mesure de la déflexion de ces poutres, un film d'AlN de $0,8 \mu \mathrm{m}$ d'épais a été déposé dans les mêmes conditions que celles des microsystèmes présentées précédemment.

\subsection{Mesures interférométriques}

Aussi bien les structures tests que les microsystèmes ont été caractérisés en utilisant une plateforme basée sur un interféromètre de type Twyman-Green et dont les performances ont été préalablement validées [16,17]. La figure 3 présente un schéma de la plateforme. Cette technique permet des mesures en statique et en dynamique. L'utilisation d'un module de contrôle thermique (module Peltier) permet de fixer la température entre $15{ }^{\circ} \mathrm{C}$ et $60{ }^{\circ} \mathrm{C}$ avec une précision de $0,01^{\circ} \mathrm{C}$. Les mesures statiques consistent en l'évaluation de la déflexion des poutres lorsqu'aucune tension ou une tension continue (entre $\pm 15 \mathrm{~V}$ ) est appliquée entre les électrodes. Cinq interférogrammes avec un décalage relatif de phase de $90^{\circ}$ sont enregistrés

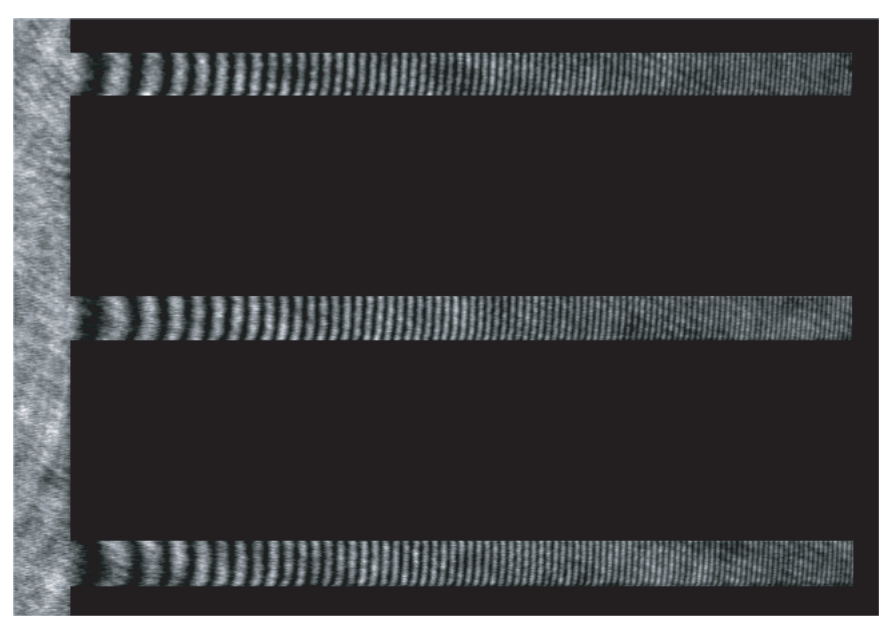

[um]

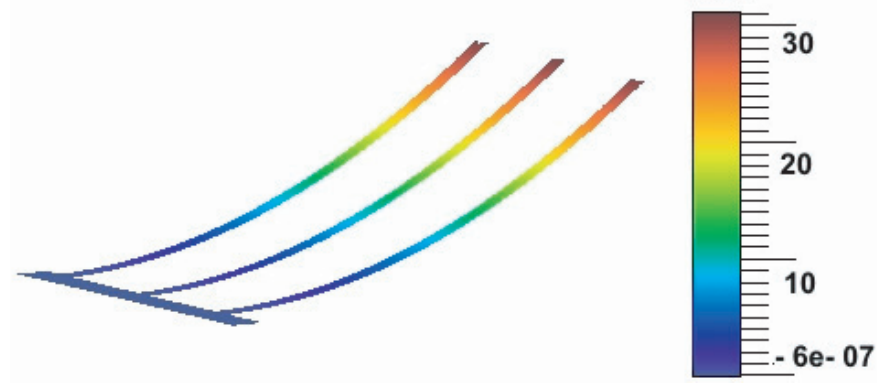

Fig. 4. Exemple d'interférogramme utilisé dans l'algorithme 5 images pour la mesure du profil des poutres (a) et profil 3D correspondant (b).

et ensuite traités numériquement à l'aide d'un algorithme de décalage de phase à cinq images pour déterminer de façon précise le profil complet des poutres $(20 \mathrm{~nm}$ de précision sur le déplacement vertical) [16, 17]. La figure 4a présente un exemple d'interférogramme et la figure $4 \mathrm{~b}$ de déformée reconstruite de trois poutres adjacentes de $800 \mu \mathrm{m}$ de long lorsqu'aucune tension n'est appliquée (1,4 $\mu \mathrm{m}$ d'AlN d'épais). Pour les mesures dynamiques, une tension sinusoïdale de $8 \mathrm{~V}$ crête-crête est appliquée aux bornes des électrodes et le premier mode de résonance est déterminé par la technique du temps moyenné [16]. Comme ces mesures sont utilisées pour l'estimation de grandeurs physiques, par exemple les contraintes ou le coefficient piézoélectrique, la connaissance précise de la métrologie des micropoutres est nécessaire. Ainsi, pour chacun des réseaux de poutres d'un wafer, les épaisseurs d'AlN et des électrodes ont été mesurées par profilométrie mécanique (quelques nm de précision). De même, les épaisseurs du Si ont été déterminées par MEB avec une précision de $0,2 \mu \mathrm{m}$.

\subsection{Mesures par nanoindentation}

Des essais de nanoindentation ont été réalisés sur les films d'AlN des structures tests à l'aide d'un nanoindenteur $\mathrm{II}^{S}$ (Nano-Instrument) équipé d'une pointe Berkovich. Cette étude est conduite en utilisant la technique de mesure de la rigidité en continu [18] avec une 
fréquence de $45 \mathrm{~Hz}$ pour une amplitude de vibration de $1 \mathrm{~nm}$ pendant la pénétration de l'indenteur dans l'échantillon. Ceci a permis de déterminer les valeurs du module d'indentation $M_{<\mathrm{hkl}}>$ et de la dureté $H_{\mathrm{b}<\mathrm{hkl}>}$ des films d'AIN déposés sur les électrodes de Pt ou de CrNi.

Pour chaque échantillon testé, la séquence de mesure consiste en 15 indents espacés entre eux de $50 \mu \mathrm{m}$, sur trois ou quatre zones différentes du film (soit $15 \times$ $3 / 4$ indents), avec une profondeur de pénétration maximale de $h_{\max }=200 \mathrm{~nm}$ et couvrant ainsi une aire de $200 \times 200 \mu \mathrm{m}^{2}$ pour chaque zone. La vitesse de pénétration n'est pas constante mais croissante avec la profondeur de 1 à $8 \mathrm{~nm} \cdot \mathrm{s}^{-1}$. Rappelons que l'épaisseur des films d'AlN est de $0,8 \mu \mathrm{m}$.

\subsection{Mesures par microscopie à force atomique, AFM}

L'écart type obtenu sur les mesures de nanoindentation est fortement correlé à la rugosité de surface des films $[19,20]$. Ainsi la rugosité moyenne $R_{\mathrm{ms}}$ a été évaluée avec un AFM (PSIA, XE 150) en mode non contact. Une aire de $7 \times 7 \mu \mathrm{m}^{2}$ a été scannée sur trois endroits différents de la surface de façon à ce que ces images soient représentatives de la surface des films. On trouve pour les films d'AlN, $R_{\mathrm{ms}}=2,4 \pm 0,4 \mathrm{~nm}$ pour $\mathrm{AlN} / \mathrm{CrNi}$ et $R_{\mathrm{ms}}=2,0 \pm 0,3$ pour AlN/Pt.

\subsection{Mesures de diffraction des R.X.}

On utilise la raie $K_{\alpha}$ du $\mathrm{Cu}$ sur un système X'Pert MPD PANalytical pour acquérir les informations $\theta-2 \theta$ dans une configuration Bragg-Brentano avec un pas de $0,02^{\circ}(2 \theta)$ et un pas de temps de 5 s. Les films d'AlN étant hautement texturés, des $\Omega$ scans ont été réalisés sur la raie de diffraction (002) de l'AlN, à $\theta=18^{\circ}$ avec un pas de $0,04^{\circ}(2 \theta)$ pour un pas de temps de 2 s et un $\Delta \Omega=20^{\circ}$. Pour le calcul de la largeur une simple fonction est extraite des données brutes par déconvolution avec une résolution de $\delta \theta=0,01^{\circ}$.

\section{Equations pour les poutres multimorphes}

Depuis les premiers travaux de Stoney en 1909 [21] plusieurs modèles théoriques traitant de l'état de contrainte dans un film mince déposé sur un substrat isotrope épais ont été publiés [22-24]. Les modèles relatifs aux films multimorphes élastiques sont apparus depuis les années 80. De plus, le comportement d'un bimorphe piézoélectrique sous l'action d'un champ électrique a été analysé en détail par Smits et Choi [25], puis l'extension à des structures et des configurations plus complexes a été réalisée par quelques auteurs [26-28].

Dans des travaux précédents [29,30], à l'aide de la théorie des composites stratifiés (CLT) appliquée à une poutre composée de trois films dont un piézoélectrique avec un comportement linéaire, les relations entre la déflexion de la structure en fonction des contraintes appliquées ou résiduelles, de la tension appliquée ou de la température ont été établies. Ce sont ces relations qui seront appliquées dans le présent article (Éqs. (1) à (8)).

\subsection{Contrainte résiduelle liée à la déflexion de la poutre}

L'utilisation du même métal pour les électrodes inférieure et supérieure et leur faible épaisseur a permis de simplifier le problème en considérant que la poutre en $\mathrm{Si}$ ne supporte que deux films; un film d'AlN et un film d'électrode en CrNi dont l'épaisseur est la somme des épaisseurs des deux électrodes. On traite donc le problème d'un trimorphe. Les contraintes dans l'AlN et le film métallique créent une déflexion mécanique $\delta_{\mathrm{m}}$ de la poutre telle que :

$$
\delta_{\mathrm{m}}=\frac{3(1-\nu)}{E_{\mathrm{eq}} h_{\mathrm{eq}}^{2}} \frac{E_{\mathrm{s}} h_{\mathrm{s}}^{2}-\left(E_{\mathrm{f}} h_{\mathrm{f}}+E_{\mathrm{e}} h_{\mathrm{e}}\right)\left(h_{\mathrm{e}}+h_{\mathrm{f}}\right)}{E_{\mathrm{s}} h_{\mathrm{s}}+E_{\mathrm{f}} h_{\mathrm{f}}+E_{\mathrm{e}} h_{\mathrm{e}}} L^{2} \sigma_{0}
$$

avec la contrainte globale $\sigma_{o}$ :

$$
\sigma_{0}=\frac{\sigma_{\mathrm{f}} h_{\mathrm{f}}+\sigma_{\mathrm{e}} h_{\mathrm{e}}+\sigma_{\mathrm{s}} h_{\mathrm{s}}}{h_{\mathrm{eq}}}
$$

et où $E_{\mathrm{i}}, \mathrm{h}_{\mathrm{i}}$ et $\sigma_{\mathrm{i}}$ avec $\mathrm{i}=\mathrm{s}, \mathrm{f}$, e sont le module d'Young, l'épaisseur et la contrainte dans le substrat en Si (s), le film d'AlN (f) et le film d'électrode (e). $L$ représente la longueur de la poutre et $\nu$ le coefficient de Poisson considéré comme identique pour les trois matériaux. L'épaisseur équivalente $h_{\mathrm{eq}}$ est égale à la somme de $h_{\mathrm{f}}, h_{\mathrm{s}}$ et $h_{\mathrm{e}}$ alors que le module équivalent $E_{\text {eq }}$ est donné par :

$$
E_{\mathrm{eq}}=\frac{K_{1}+K_{2}+K_{3}+K_{4}+K_{5}}{\left(E_{\mathrm{f}} h_{\mathrm{f}}+E_{\mathrm{s}} h_{\mathrm{s}}+E_{\mathrm{e}} h_{\mathrm{e}}\right) h_{\mathrm{eq}}^{3}}
$$

avec

$$
\begin{aligned}
& K_{1}=E_{\mathrm{f}}^{2} h_{\mathrm{f}}^{4}+E_{\mathrm{s}}^{2} h_{\mathrm{s}}^{4}+E_{\mathrm{e}}^{2} h_{\mathrm{e}}^{4} \\
& K_{2}=2 E_{\mathrm{s}} E_{\mathrm{e}} h_{\mathrm{s}} h_{\mathrm{e}}\left(2 h_{\mathrm{s}}^{2}+2 h_{\mathrm{e}}^{2}+3 h_{\mathrm{s}} h_{\mathrm{e}}\right) \\
& K_{3}=2 E_{\mathrm{f}} E_{\mathrm{e}} h_{\mathrm{f}} h_{\mathrm{e}}\left(2 h_{\mathrm{f}}^{2}+2 h_{\mathrm{e}}^{2}+3 h_{\mathrm{f}} h_{\mathrm{e}}\right) \\
& K_{4}=2 E_{\mathrm{f}} E_{\mathrm{s}} h_{\mathrm{f}} h_{\mathrm{s}}\left(2 h_{\mathrm{s}}^{2}+2 h_{\mathrm{f}}^{2}+3 h_{\mathrm{s}} h_{\mathrm{f}}\right) \\
& K_{5}=12 E_{\mathrm{s}} E_{\mathrm{f}} h_{\mathrm{s}} h_{\mathrm{f}} h_{\mathrm{e}} h_{\mathrm{eq}}
\end{aligned}
$$

Il est intéressant de noter que le développement au premier ordre des équations (3), considérant $h_{\mathrm{e}}=0$ et $\sigma_{s}=0$ dans les équations (1)-(3), donne une généralisation de l'équation de Stoney :

$$
\sigma_{\mathrm{f}}=\frac{E_{\mathrm{s}} h_{\mathrm{s}}^{2} \delta_{\mathrm{m}}}{3(1-\nu) h_{\mathrm{f}} L^{2}}[1+4 \underbrace{\frac{E_{\mathrm{f}} h_{\mathrm{f}}}{E_{\mathrm{s}} h_{\mathrm{s}}}}_{\mathrm{A}}(1+\underbrace{\frac{3 h_{\mathrm{f}}}{2 h_{\mathrm{s}}}}_{\mathrm{B}})]
$$

L'équation (4) est en bon accord avec la relation établie par Röll [20], seul le terme $B$ diffère. Dans notre cas particulier, l'utilisation de l'équation de Stoney aurait introduit une sous-estimation conséquente de la contrainte dans le film puisque les valeurs des termes $A$ et $B$ sont 0,78 et 0,11 . Dans notre cas $E_{\mathrm{f}} / E_{\mathrm{s}}=2,6$ et $h_{\mathrm{f}} / f_{\mathrm{s}}=$ $7,510 \times 10^{-2}$. 


$$
\delta_{\mathrm{Th}}=3 \frac{\left[E_{\mathrm{s}} h_{\mathrm{s}}^{2}-\left(E_{\mathrm{f}} h_{\mathrm{f}}+E_{\mathrm{e}} h_{\mathrm{e}}\right)\left(h_{\mathrm{e}}+h_{\mathrm{f}}\right)\right]\left[\alpha_{\mathrm{s}}\left(E_{\mathrm{f}} h_{\mathrm{f}}+E_{\mathrm{e}} h_{\mathrm{e}}\right)-\left(E_{\mathrm{f}} \alpha_{\mathrm{f}} h_{\mathrm{f}}+E_{\mathrm{e}} \alpha_{\mathrm{e}} h_{\mathrm{e}}\right)\right]}{E_{\mathrm{eq}} h_{\mathrm{eq}}^{3}\left(E_{\mathrm{f}} h_{\mathrm{f}}+E_{\mathrm{s}} h_{\mathrm{s}}+E_{\mathrm{e}} h_{\mathrm{e}}\right)} L^{2} \Delta T
$$

\subsection{Déflexion induite par la piézoélectricité et premier mode de résonance}

Pour une tension appliquée $V$, les propriétés piézoélectriques linéaires de l'AlN génèrent une déflexion supplémentaire $\delta_{p}$ qui est reliée au coefficient piézoélectrique $d_{31}$ par :

$$
\delta_{p}=-\frac{3}{E_{\mathrm{eq}} h_{\mathrm{eq}}^{2}} \frac{E_{\mathrm{f}}\left(E_{\mathrm{s}} h_{\mathrm{s}}+E_{\mathrm{e}} h_{\mathrm{e}}\right)}{E_{\mathrm{s}} h_{\mathrm{s}}+E_{\mathrm{f}} h_{\mathrm{f}}+E_{\mathrm{e}} h_{\mathrm{e}}} d_{31} L^{2} V
$$

Cette expression est en accord avec celle développée par Wang et al. [26] pour une poutre symétrique à trois films. En posant $h_{\mathrm{e}}=0$ dans l'équation précédente on retrouve aisément l'équation classique pour un film piézoélectrique déposé sur une poutre épaisse [25, 29,30].

Le premier mode de résonance pour un multimorphe s'écrit :

$$
f_{1}=\frac{p_{1}^{2}}{4 \pi \sqrt{3}} \frac{h_{\mathrm{eq}}}{L^{2}} \sqrt{\frac{E_{\mathrm{eq}}}{\rho_{\mathrm{eq}}}}
$$

où $h_{\mathrm{eq}}$ est l'épaisseur équivalente et $E_{\mathrm{eq}}$ le module équivalent défini précédemment (Éq. (3)). Le terme $p_{1}$ vaut 1,875 et $\rho_{\mathrm{eq}}$ est la masse volumique équivalente définie par :

$$
\rho_{\mathrm{eq}}=\frac{\rho_{\mathrm{s}} h_{\mathrm{s}}+\rho_{\mathrm{f}} h_{\mathrm{f}}+\rho_{\mathrm{e}} h_{\mathrm{e}}}{h_{\mathrm{eq}}}
$$

\subsection{Influence de la température}

Sous un chargement thermique $\Delta T$, la déflexion correspondante de la poutre $\delta_{\mathrm{Th}}$, fonction des coefficients de dilatation des trois films, $\alpha_{\mathrm{s}}, \alpha_{\mathrm{f}}, \alpha_{\mathrm{e}}$, est donnée par :

$$
\text { voir équation (8) ci-dessus. }
$$

\section{Résultats et interprétations}

\subsection{Résultats XRD}

La figure 5 montre les spectres de diffraction $\mathrm{X}$ des films d'AlN déposés sur les électrodes en $\mathrm{CrNi}$ et $\mathrm{Pt}$. Les cristallites du film d'AlN sont parfaitement orientées (002) pour l'électrode en $\mathrm{CrNi}$, l'axe $\vec{c}$ de la structure hexagonale est perpendiculaire à la surface du substrat. Les différents pics du CrNi sont identifiés. Cependant, la présence de pics additionnels (notés avec une étoile sur la Fig. 5) correspond à des composés de l'électrode non identifiés, certainement en relation avec la nature de la cible (oxy-nitrure de CrNi?). Pour l'électrode de Pt, orientée (111), la direction (002) des cristallites est toujours la plus prononcée mais un pic assez large au voisinage de $45^{\circ}$ semble révéler la présence d'une partie amorphe.

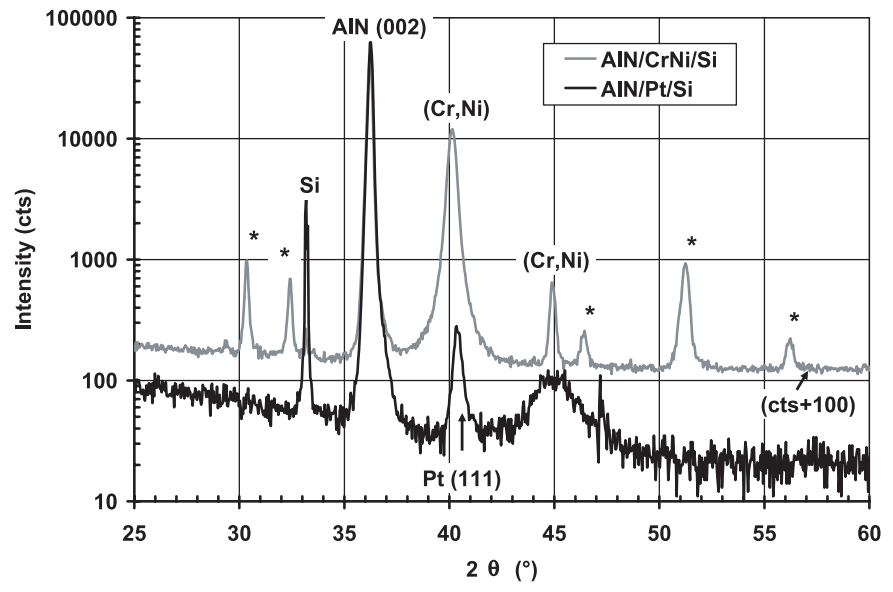

Fig. 5. Diagrammes de diffraction $\mathrm{X}$ des films d'AlN sur $\mathrm{CrNi}$ et Pt.

Dans ce cas, la morphologie du film d'AlN est plus complexe; des cristallites bien orientées (002) côtoyant des parties plus ou moins amorphes. La $F W H M$ du scan $\Omega$ du plan (002) de l'AlN est plus petit pour l'électrode de Pt $\left(1,37^{\circ}\right)$ que pour celle en $\mathrm{CrNi}\left(2,68^{\circ}\right)$, ce qui indique une meilleure orientation dans le cas d'une électrode de Pt. Ces différentes observations, en relation avec les conditions de déposition mentionnées précédemment sont en bon accord avec les résultats de Ishihara et al. [10].

L'électrode $\mathrm{CrNi}$ semble meilleure pour les applications MEMS puisque dans ce cas, l'AlN ne présente qu'une seule orientation (002) très bien définie puisque $F W H M=2,68^{\circ}$.

\subsection{Valeurs des modules d'indentation}

En nanoindentation, le module $M_{<\mathrm{hkl}}>$ mesuré sur un monocristal dont la normale à la surface a pour cosinus directeurs $\alpha_{\mathrm{i}}$ est donné par [31,32] :

$$
\begin{aligned}
& M_{<\mathrm{hkl}>}=16 \pi^{2}\left[\int_{0}^{2 \pi} \alpha_{\mathrm{m}} \beta_{\mathrm{km}}^{-1}\left(C_{i j} \gamma\right) \alpha_{\mathrm{k}} \mathrm{d} \gamma\right]^{-1} \\
& \text { avec } M_{<\mathrm{hkl}>}=\left[\frac{1}{M_{\mathrm{r}}}-\left(\frac{1-\nu^{2}}{E}\right)_{\mathrm{ind}}\right]^{-1} \text { and } \\
& M_{\mathrm{r}}=\frac{\sqrt{\pi}}{2 \beta \sqrt{A}} \cdot \frac{\mathrm{d} P}{\mathrm{~d} h} A\left(h_{\mathrm{c}}\right)=24,5 h_{\mathrm{c}}^{2}+\sum_{\mathrm{n}=1}^{4}\left(a_{\mathrm{n}} h_{\mathrm{c}}^{1 / \mathrm{n}}\right), \\
& h_{\mathrm{c}}=h-\varepsilon\left(\frac{P}{S}\right)
\end{aligned}
$$

$A$ est l'aire de contact projetée, $P$ la charge appliquée, $S=(\mathrm{d} P / \mathrm{d} h)$ la rigidité à la décharge mesurée à la profondeur d'indentation $h, \beta=1,034$ et $\varepsilon=0,72$ pour une pointe Berkovich. $\left(E / 1-\nu^{2}\right)$ ind est le module réduit du 
Tableau 1. Valeurs des rigidités élastiques et des modules d'indentation $M_{<\mathrm{hkl}>}$.

\begin{tabular}{lcccccccc}
\hline & $C_{11}$ & $C_{12}$ & $C_{33}$ & $C_{44}$ & $C_{66}$ & $M_{<001>}$ & $M_{<100>}$ & Ref. \\
\hline Pulvérisé Haute Qualité & 360 & 123 & 410 & 116 & 119 & 342 & 319 & {$[35]$} \\
Faible Qualité & 326 & 122 & 401 & 99 & 108 & 316 & 286 & {$[35]$} \\
Épitaxié & 345 & 120 & 395 & 118 & 110 & 335 & 306 & {$[36]$} \\
Volumique & 396 & 108 & 373 & 116 & 129.5 & 329 & 344 & {$[37]$} \\
\hline
\end{tabular}

diamant de l'indenteur, $\gamma$ l'angle dans le plan de la surface et $\beta_{k m}\left(C_{i j}, \gamma\right)$ une matrice très compliquée fonction des constantes élastiques $C_{i j}$ et de $\gamma[31,32]$. Dans ce cas, en fonction de l'orientation des cristallites se produisant durant la phase de dépôt, le module $M_{<\mathrm{hkl}>}$ ne doit pas être constant.

La dureté mesurée est donnée par :

$$
H_{\mathrm{b}<\mathrm{hkl}>}=\frac{P}{A}
$$

En général il n'existe pas de solution analytique à l'équation (9). Cependant, pour quelques configurations, quelques solutions très proches des résultats numériques résultant de l'équation (9) sont disponibles. Ainsi, pour la symétrie hexagonale qui est le cas de l'AlN (isotropie transverse : $\left.C_{66}=\left(C_{11}-C_{12}\right) / 2\right)$; on montre que [33] :

$$
\begin{gathered}
M_{<001>}=\frac{2}{\sqrt{\frac{C_{11}}{C_{11} C_{33}-C_{13}^{2}}\left(\frac{1}{C_{44}}+\frac{2}{\sqrt{C_{11} C_{33}}+C_{13}}\right)}} \\
M_{<100>}=M_{<110>}=\sqrt{\frac{C_{11}^{2}-C_{12}^{2}}{C_{11}} \sqrt{\frac{C_{11}}{C_{33}}} M_{<001>}}
\end{gathered}
$$

Entre ces deux valeurs il n'existe pas de solution analytique, seulement des solutions numériques approchées [34]. Différentes valeurs des constantes élastiques $C_{i j}$ sont disponibles pour l'AlN volumique [35-37], déposé par pulvérisation cathodique [35] et pour une croissance épitaxiale à haute température [36,37]. Il a été montré que les valeurs de ces constantes pour les films pulvérisés dépendent d'une façon non négligeable de la microstructure des films elle-même fortement affectée par les conditions de déposition. Le tableau 1 donne les valeurs des $C_{i j}$ et celles de $M_{<001>}$ and $M_{<100>}$ calculées avec les équations (11), (12).

Les figures $6 \mathrm{a}$ et $\mathrm{b}$ représentent les évolutions des valeurs moyennes du module $M_{<\mathrm{hkl}>}$ et de la dureté $H_{\mathrm{b}<\mathrm{hkl}>}$ en fonction de la profondeur réduite d'indentation $h / h_{\mathrm{f}}$ pour les deux types d'électrode $(\mathrm{CrNi} / \mathrm{Si}$ and $\mathrm{Pt} / \mathrm{Si}$ ) ainsi que pour l'AlN sur le verre [38]. Pour étudier l'évolution de ces paramètres avec la morphologie de la microstructure des films déposés, tout en prenant compte de la présence du substrat, il est nécessaire d'analyser les résultats pour les faibles profondeurs d'indentation, typiquement pour $h / h_{\mathrm{f}} \leqslant 10 \%[39,40]$. Ainsi, on étudie les réponses pour $h=25,50$ and $75 \mathrm{~nm}(3,1<$ $\left.h / h_{\mathrm{f}}<9,4 \%\right)$. Le module d'indentation est une fonction décroissante de $h / h_{\mathrm{f}}$ (Fig. 6a) puisque $M_{<\mathrm{hkl}}>(\mathrm{AlN})>$ $M_{<100>}(\mathrm{Si})=189 \mathrm{GPa}$. Ceci est également vrai pour le substrat verre puisque $M\left(\mathrm{Si}_{2}\right)=80 \mathrm{GPa}$. Pour $h / h_{\mathrm{f}} \cong 0$, (a)

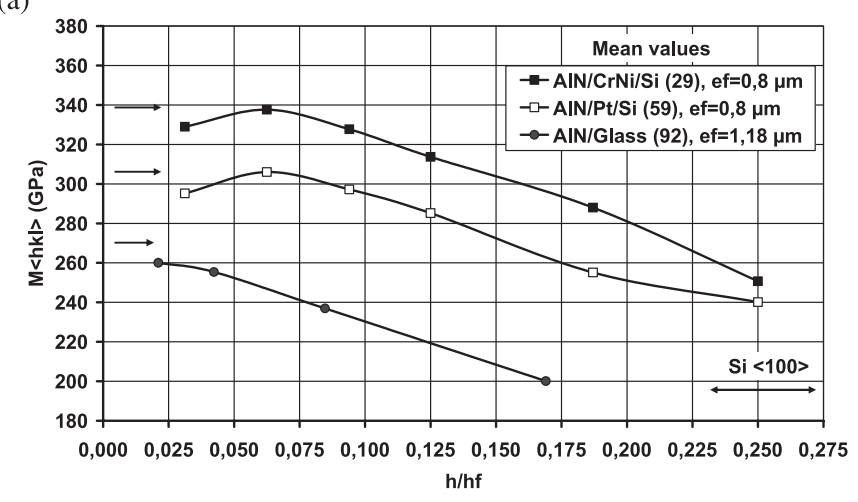

(b)

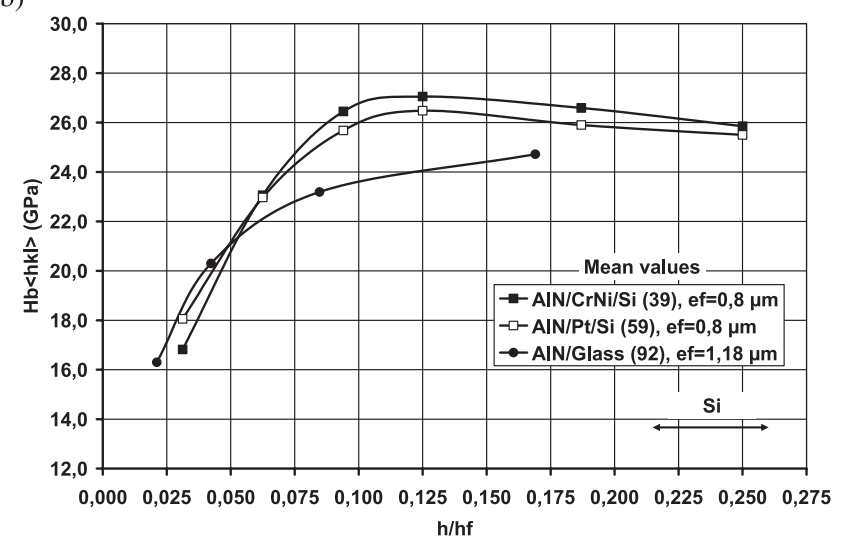

(c)

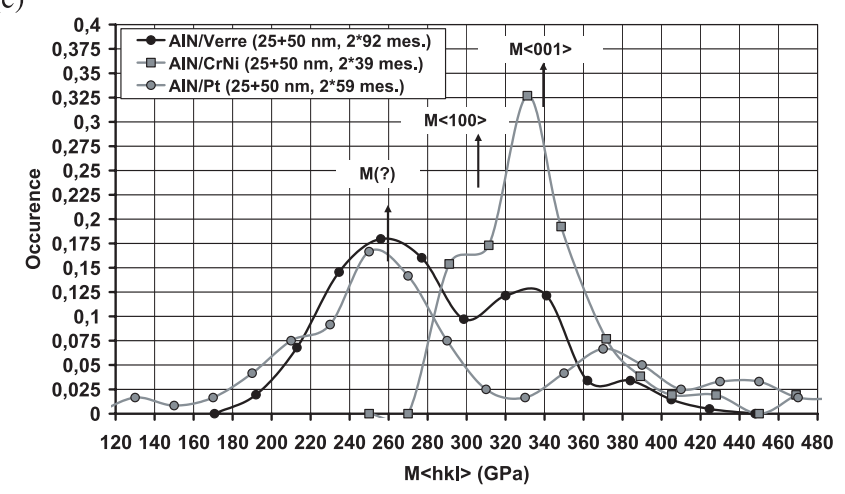

Fig. 6. Évolution du module d'indentation $M_{<\mathrm{hkl}>}$ (a), de la dureté $H_{\mathrm{b}<\mathrm{hkl}>}(\mathrm{b})$ en fonction de la profondeur réduite d'indentation pour l'AlN déposé sur des substrats en Pt, CrNi et verre. Histogrammes des valeurs de $M_{<\mathrm{hkl}>}$ (c).

les valeurs moyennes calculées sont $M_{<\mathrm{hkl}>}=340 \pm$ $10 \mathrm{GPa}$ pour $\mathrm{AlN} / \mathrm{CrNi} / \mathrm{Si}, M_{<\mathrm{hkl}>}=305 \pm 10 \mathrm{GPa}$ for 
AlN/Pt/Si et $M_{<\text {hkl }>}=270 \pm 10 \mathrm{GPa}$ pour AlN/verre. Concernant la dureté (Fig. 6b), $H_{\mathrm{b}<\mathrm{hkl}>}$ est une fonction d'abord croissante de la profondeur réduite $h / h_{\mathrm{f}} \leqslant 10 \%$ et ensuite légèrement décroissante puisque la dureté du silicium est inférieure à celle des films, $H_{\mathrm{b}}(\mathrm{AlN})>$ $H_{\mathrm{B}}(\mathrm{Si})=13,5 \mathrm{GPa}$. Cependant, comme l'a montré Xue et al. [41], pour les faibles profondeurs d'indentation, la forme sphérique de la pointe de l'indenteur dont le rayon est estimé à $400 \mathrm{~nm}$ dans la présente étude, conduit à une décroissance de la dureté pour les très faibles profondeurs d'indentation. Ainsi, seules les valeurs pour $h / h_{\mathrm{f}} \geqslant 10 \%$ peuvent être considérées comme celles de la dureté Berkovich des films. Pour les trois types de substrat, la valeur moyenne de la dureté des films est de $H_{\mathrm{b}<\mathrm{hkl}>}=$ $26,0 \pm 1,5 \mathrm{GPa}$.

Cependant, l'écart type relatif $\sigma_{\mathrm{E}} / E_{\text {moy }}\left(\right.$ et $\left.\sigma_{H} / H_{\text {moy }}\right)$ où $E_{\text {moy }}$ est la valeur moyenne du module d'Young mesuré sur un matériau isotrope et $\sigma_{\mathrm{E}}$ l'écart type déterminé sur plus de 25 mesures [19] est relié au paramètre adimensionnel $R_{\mathrm{ms}} / h$ par la relation $[19,20]$ :

$$
\frac{\sigma_{E}}{E_{\mathrm{moy}}}=\alpha\left(\frac{R_{\mathrm{ms}}}{h}\right)^{m}
$$

Pour de nombreux matériaux déposés par pulvérisation, les valeurs de $\alpha$ et $m$ ont été identifiées; $\alpha=0,346$ et $m=0,64$ [20]. Si l'on prend les valeurs mesurées de $R_{\mathrm{ms}}$ $\left(2,0<R_{\mathrm{ms}}<2,4 \mathrm{~nm}\right)$, le rapport $\frac{\sigma_{\mathrm{E}}}{E_{\text {mean }}}=\frac{\sigma_{\mathrm{E}}}{\left(1-\nu^{2}\right) M_{<\mathrm{hkl}}}$ (avec $\nu=0,25$ ) devrait être de $7 \%$ et $5 \%$ respectivement pour $h=25 \mathrm{~nm}$ et $50 \mathrm{~nm}$. Expérimentalement, on obtient $11 \%$ et $7 \%$ pour $\mathrm{AlN} / \mathrm{CrNi}, 18 \%$ et $12 \%$ pour AlN/verre et $35 \%$ et $24 \%$ pour AlN /Pt. Ces valeurs sont supérieures à celles estimées avec l'équation (13), le facteur multiplicatif est d'environ 1,4, 2,4 et 5,2 respectivement. Comme il l'a été montré sur des films minces de PZT $[42,43]$ ce facteur peut être considéré comme un indicateur du degré de désorientation des cristallites. Ceci est en accord avec les spectres XRD (Fig 5) : pour les cristallites bien orientées (002) pour AlN/CrNi il y a un assez bon accord entre les valeurs expérimentale et calculée, le rapport n'est que de 1,4, alors que pour les films plus polycristallins, AlN/verre [38] et AlN/Pt où les cristallites orientées (002) sont plus ou moins entourées de phase amorphe, ce facteur est égal à 2,4 et 5,2 respectivement. Pour analyser la signification de ce facteur les histogrammes des valeurs mesurées de $M_{<\mathrm{hkl}}>$ pour $h=25$ et $50 \mathrm{~nm}$ et pour chaque type de film sont reportés sur la figure 6c. Pour les cristallites bien orientées (002), le cas de AlN/CrNi (facteur 1,4) il n'apparaît qu'un seul pic centré à $M_{<\mathrm{hkl}>}=332 \mathrm{GPa}$, proche de la valeur moyenne, et correspondant à $M_{<001>}$ calculé avec les valeurs des $C_{i j}$ pour les films pulvérisés de haute qualité $\left(M_{<001>}=342 \mathrm{GPa}\right)$ et épitaxiés $\left(M_{<001>}=335 \mathrm{GPa}\right)$, tableau 1 .

Cependant, pour l'AlN sur verre (facteur 2,4 et valeur moyenne égale à $270 \mathrm{GPa}$ ) il y a deux pics bien définis, le premier centré à $M_{<\mathrm{hkl}>}=335 \mathrm{GPa}$ et correspondant à l'orientation (002), comme pour $\mathrm{AlN} / \mathrm{CrNi}$ et le second à $M_{<\mathrm{hkl}}>260 \mathrm{GPa}$. Pour AlN/Pt (facteur 5,2 et valeur moyenne égale à $305 \mathrm{GPa}$ ) il y a aussi deux pics, à $370 \mathrm{GPa}$ et $260 \mathrm{GPa}$. Le premier (370 GPa)

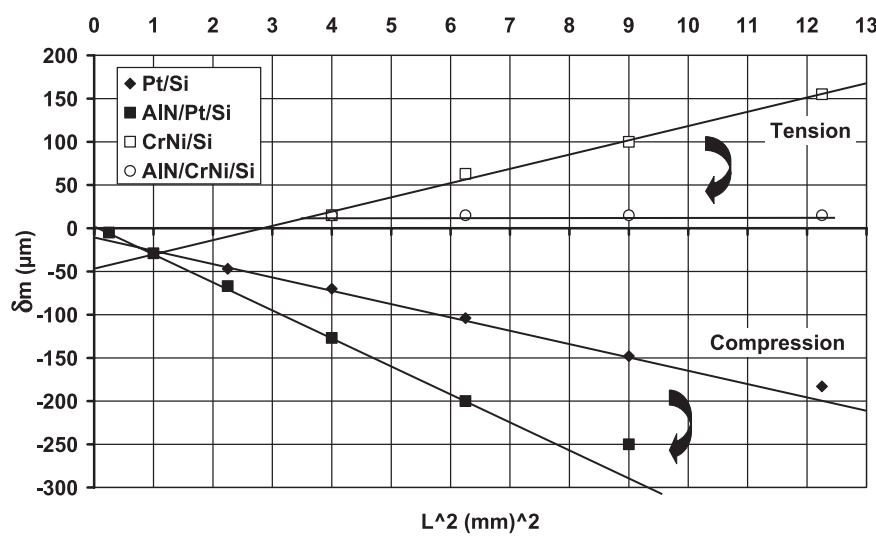

Fig. 7. Mesure de la déflexion des poutres tests avant et après déposition de l'AlN en fonction du carré de leur longueur.

est certainement dû aux cristallites extrêmement bien orientées selon (002). Concernant le pic à $260 \mathrm{GPa}$, soit $E=244 \mathrm{GPa}$ pour $\nu=0,25$, et la valeur correspondante de la dureté $H_{\mathrm{b}<\mathrm{hkl}>}=17 \mathrm{GPa}$, ces valeurs semblent trop élevées pour être attribuées à une phase amorphe : $E \cong 140 \mathrm{GPa}$ et $H_{\mathrm{b}}=9,2 \mathrm{GPa}[44]$ et $80<E<220 \mathrm{GPa}$ pour $3,5<H_{\mathrm{b}}<20 \mathrm{GPa}$ [45]. Cependant, ces valeurs sont plus en accord avec celles de la phase cristalline $\mathrm{Al}$ ON obtenue sur des substrats de Si et de verre à haute température : $E=261 \mathrm{GPa}, H_{\mathrm{b}}=17,8 \mathrm{GPa}$ [44]. À noter que l'absence de cette phase pour l'électrode en CrNi est peut-être due à l'oxydation de l'électrode comme on peut l'observer sur le spectre XRD, figure 5a. La dureté Berkovich associée à l'orientation (002) est d'environ $H_{\mathrm{b}(002)}=25 \pm 2 \mathrm{GPa}$. Entre les deux types d'électrodes testées, le CrNi semble le meilleur choix puisqu'il conduit à des cristallites bien orientées (002) et à un module d'indentation $M_{<001>}$ très bien défini. La valeur du module d'Young calculée à partir des valeurs des $C_{i j}$ [35] est égale à $333 \mathrm{GPa}$ dans la direction $\vec{c}$, proche de $M_{<001>}$ et de $284 \mathrm{GPa}$ dans une direction perpendiculaire.

\subsection{Valeurs des contraintes résiduelles dans les films minces}

La flexion des poutres des structures tests avant et après déposition de l'AlN sur les électrodes en $\mathrm{CrNi}$ et $\mathrm{Pt}$ donne des informations sur les niveaux des contraintes dans les électrodes et le film lui-même. Comme le montre la figure 7 , déflexion mécanique $\delta_{\mathrm{m}}$ fonction du carré $L^{2}$ de la longueur des poutres, la contrainte est tensive pour le film de $\mathrm{CrNi}$ et compressive pour le Pt. Le changement de pente après déposition de l'AlN indique que la contrainte est compressive quel que soit le type d'électrode. L'évaluation numérique à l'aide des équations (1) à (3) avec $\sigma_{\mathrm{si}}=0, E_{\mathrm{Si}}=129 \mathrm{GPa}$, $E_{\mathrm{AlN}}=330 \mathrm{GPa}, E_{\mathrm{CrNi}}=237 \mathrm{GPa}$ et $E_{\mathrm{Pt}}=168 \mathrm{GPa}$ avec $\nu=0,3$, donne $\sigma_{\mathrm{CrNi}}=+1110 \mathrm{MPa}$ pour le film de $\mathrm{CrNi}$ et $\sigma_{\mathrm{Pt}}=-700 \mathrm{MPa}$ pour le film de Pt. Après la déposition de l'AlN, en tenant compte des valeurs de contrainte dans les électrodes et en appliquant à nouveau 
(a)

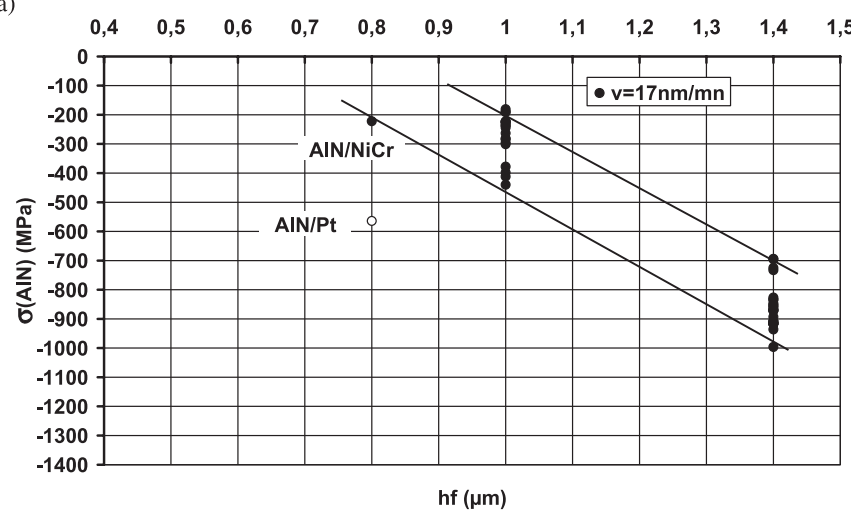

(b)

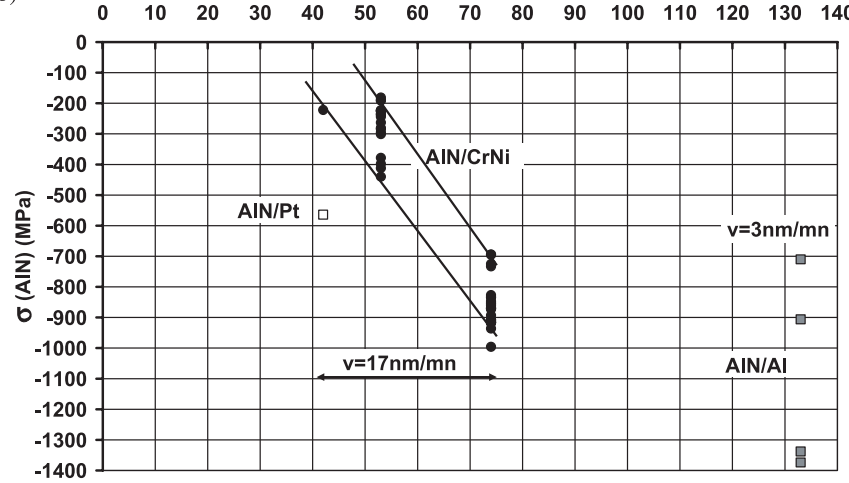

Time of Sputtering (mn)

Fig. 8. Contrainte calculée dans le film d'AlN en fonction de son épaisseur (a) et du temps de déposition (b).

les équations (1) à (3), on calcule $\sigma_{\mathrm{AlN}}=-220 \mathrm{MPa}$ pour $\mathrm{AlN} / \mathrm{CrNi}$ et $\sigma_{\mathrm{AlN}}=-564 \mathrm{MPa}$ pour AlN/Pt.

Le $\mathrm{CrNi}$ conduit donc à un niveau de contrainte plus faible qu'avec le Pt. De plus, si les épaisseurs des films CrNi et AlN sont bien choisies, il est possible d'obtenir une structure non défléchie $\delta_{\mathrm{m}}=0$ avec une contrainte globale voisine de zéro, ce qui est presque le cas reporté sur la figure 7 .

La contrainte compressive de l'AlN est, d'après la littérature [16], directement liée à la puissance DC du réacteur; plus elle augmente plus la contrainte est compressive. W.J. Meng [46] a aussi rapporté que la contrainte était une fonction de l'épaisseur du film, son amplitude présente un minimum pour une épaisseur comprise entre $0,5 \mu \mathrm{m}$ et $1 \mu \mathrm{m}$ puis croît ensuite avec l'épaisseur. Ces observations sont confirmées dans cette étude. En effet, la figure 8a montre les contraintes calculées en fonction des épaisseurs des films, non seulement pour les structures tests mais également sur les poutres des microsystèmes avec des épaisseurs d'AlN de $1 \mu \mathrm{m}$ et $1,4 \mu \mathrm{m}$. La contrainte compressive dans l'AlN croît en valeur absolue avec l'épaisseur du film.

Cependant, dans la présente étude, tous les films ont été déposés avec la même vitesse $\cong 17 \mathrm{~nm} \cdot \mathrm{min}^{-1}$ ce qui rend impossible de distinguer l'effet de l'épaisseur de celui du temps de pulvérisation. Durant la phase d'optimisation du procédé, quelques expériences ont été réalisées sur

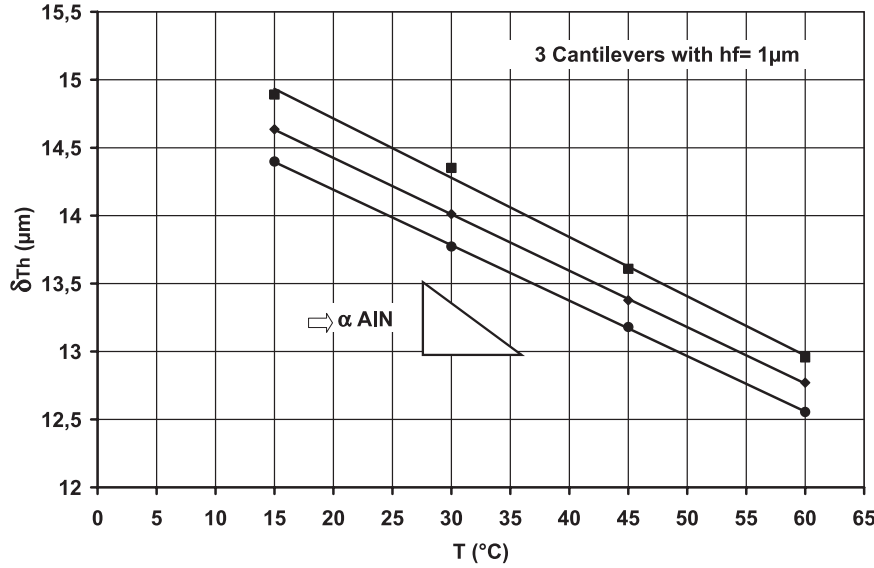

Fig. 9. Déflexion statique en fonction de la température pour trois poutres de $800 \mu \mathrm{m}$ de long.

de l'AlN déposé sur une électrode d'Al [48]. La puissance était de $150 \mathrm{~W}$ donnant une faible vitesse de déposition de l'ordre de $3 \mathrm{~nm} \cdot \mathrm{min}^{-1}$. L'épaisseur finale des films était de $0,4 \mu \mathrm{m}$. La figure $8 \mathrm{~b}$ montre, pour toutes les poutres testées, la dépendance de la contrainte $\sigma_{\text {AlN }}$ avec le temps de pulvérisation. Elle semble être une fonction croissance du temps de pulvérisation ce qui indique une forte dépendance entre la structure du film, sa cristallinité et son mode de croissance, en accord avec les résultats d'Auger et al. [14].

\subsection{Détermination du coefficient de dilatation}

Le module de contrôle thermique (module Peltier) permet de fixer la température des microsystèmes entre $15{ }^{\circ} \mathrm{C}$ et $60{ }^{\circ} \mathrm{C}$ et ainsi déterminer le coefficient de dilatation des films d'AlN (Éq. (8)). La figure 9 montre les variations de la déflexion totale $\delta_{\mathrm{m}}+\delta_{T h}$ en fonction de la température pour trois poutres différentes. L'application de l'équation (8) connaissant la pente des courbes $\left(\Delta \delta_{\mathrm{Th}} / \Delta T\right)$ et les paramètres matériaux $\left(h_{\mathrm{f}}=\right.$ $1 \mu \mathrm{m}, h_{s}=16,05 \mu \mathrm{m}, h_{\mathrm{e}}=0,506 \mathrm{~nm}, L=800 \mu \mathrm{m}$, $\alpha_{\mathrm{s}}=2,44 \times 10^{-6} \mathrm{~K}^{-1}, \alpha_{\mathrm{e}}=9,5 \times 10^{-6} \mathrm{~K}^{-1}$ ) permet d'accéder à $\alpha_{\mathrm{f}}=\alpha_{\mathrm{AlN}}$. On obtient $4,3 \times 10^{-6}<\alpha_{\mathrm{AlN}}<$ $4,6 \times 10^{-6} \mathrm{~K}^{-1}$, ce qui est en bon accord avec les valeurs reportées dans la littérature pour le matériau volumique : $4,4 \times 10^{-6}<\alpha_{\mathrm{AlN}}<5,3 \times 10^{-6} \mathrm{~K}^{-1}$ [48] et $4,03 \times 10^{-6}<\alpha_{\mathrm{AlN}}<4,84 \times 10^{-6} \mathrm{~K}^{-1}[49]$.

\subsection{Mesure des fréquences de résonance}

Un signal sinusoïdal ( $8 \mathrm{~V}$ crête-crête) est appliqué aux bornes des électrodes. La méthode d'interférométrie par temps moyenné permet de déterminer le premier mode de résonance $f_{1}$ des micropoutres. Comme il est montré sur la figure 10a et selon l'équation (6), $f_{1}$ est proportionnel à l'inverse du carré de la longueur des poutres et varie telle que $37 \mathrm{KHz}<f_{1}<500 \mathrm{KHz}$ pour $200 \mu \mathrm{m}$ $<L<800 \mu \mathrm{m}$. Les fréquences déterminées sur les microsystèmes $\mathrm{AlN} / \mathrm{Al} / \mathrm{Si}$ sont aussi reportées [47]. Il y a 

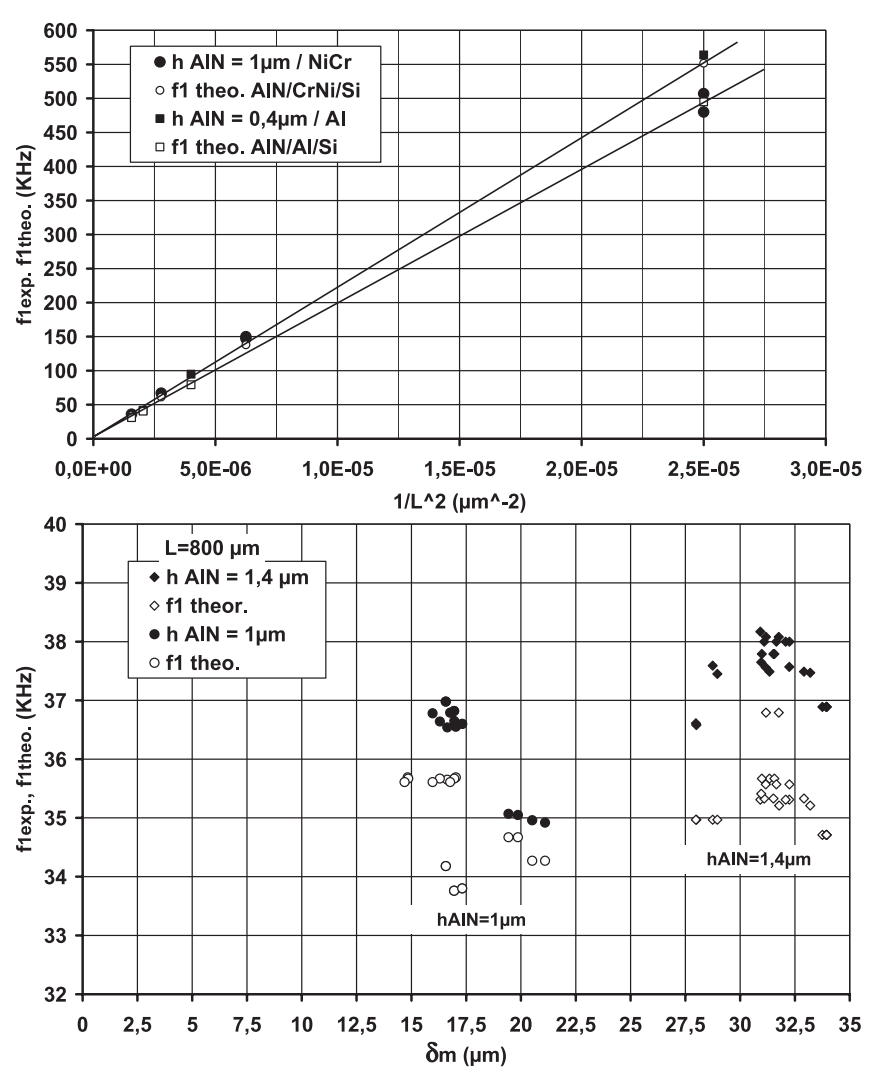

Fig. 10. Mesure de la première fréquence de résonance des micropoutres en fonction de l'inverse du carré de leur longueur (a) ou en fonction de la déflexion statique initiale pour des poutres de $800 \mu \mathrm{m}$ de long et pour des épaisseurs de $1 \mu \mathrm{m}$ et $1,4 \mu \mathrm{m}$.

un assez bon accord entre les valeurs calculées et les valeurs expérimentales (Eq. (6) et Fig. 10a), excepté pour les plus faibles longueurs où la fréquence est très haute $\left(f_{1} \cong 500 \mathrm{KHz}\right.$ pour $\left.L=200 \mu \mathrm{m}\right)$ et les incertitudes sur la géométrie exacte des poutres plus prononcées.

Une analyse plus précise pour différentes poutres de même longueur $L=800 \mu \mathrm{m}$, mais provenant du wafer $1, h_{\mathrm{f}}=1 \mu \mathrm{m}, 15$ poutres testées, et du wafer 2, $h_{\mathrm{f}}=1,4 \mu \mathrm{m}, 22$ poutres testées, a été réalisée. La figure $10 \mathrm{~b}$ présente en fonction de la déflexion initiale $\delta_{\mathrm{m}}$ les valeurs expérimentales et théoriques du premier mode $f_{1}$ en tenant compte des épaisseurs mesurées de chacun des films constituant les poutres. Les valeurs théoriques sont toujours plus faibles que les valeurs expérimentales de l'ordre de $3,1 \%$ et $6,5 \%$ pour les wafers 1 et 2 . Cette différence peut s'expliquer par la présence d'imperfections géométriques au voisinage de l'aire d'encastrement dues à un léger désalignement des deux masques durant l'attaque anisotrope du Si (étapes 5-7 de l'organigramme). Le dépassement des plans (111) du Si sous les poutres affecte la valeur réelle de la longueur d'encastrement, les valeurs réelles étant plus faibles que les valeurs mesurées sur la face supérieure des poutres. Pour obtenir les fréquences expérimentales, $L$ devrait être de l'ordre de $788 \mu \mathrm{m}(-12 \mu \mathrm{m})$ et $774 \mu \mathrm{m}(-26 \mu \mathrm{m})$ respectivement pour les wafers 1 et 2 . De plus, pour chaque wafer, l'écart relatif entre valeurs expérimentales et théoriques de $f_{1}$ varie approximativement comme $1 / L$ (voir par exemple sur la figure $\left.10 \mathrm{a}, L=200 \mu \mathrm{m}, 1 / L^{2}=2,5 \times 10^{-5} \mu \mathrm{m}^{2}\right)$ ce qui est en accord avec l'explication précédente.

\subsection{Détermination du coefficient piézoélectrique $d_{31}$}

Lorsqu'une tension continue est appliquée aux bornes des électrodes $\left( \pm 15 \mathrm{~V}\right.$ pour $h_{\mathrm{f}}=1,4 \mu \mathrm{m}$ et $\pm 10 \mathrm{~V}$ pour les films avec $\left.h_{\mathrm{f}}=1 \mu \mathrm{m}\right)$ un déplacement supplémentaire $\delta p$ (Eq. (5)) s'ajoute au déplacement mécanique $\delta m$ lié aux contraintes résiduelles (Eq. (1)). Comme on le montre sur la figure 11a, le déplacement lié à l'effet piézoélectrique est linéaire en fonction de la tension appliquée. La pente pour le wafer 2 est plus grande que pour le wafer 1 puisque l'épaisseur d'AlN est plus épaisse dans le premier cas (le Si et les électrodes ont sensiblement les mêmes épaisseurs). Cependant, en utilisant l'équation (5) et comme montré sur la figure 11 b pour différentes poutres, la valeur du coefficient $d_{31}$ pour le wafer $2\left(h_{\mathrm{f}}=1,4 \mu \mathrm{m}\right)$ est de $-2,0 \pm$ $0,15 \mathrm{pm} . \mathrm{V}^{-1}$ et de $-1,3 \pm 0,1 \mathrm{pm} . \mathrm{V}^{-1}$ pour le wafer 1 $\left(h_{\mathrm{f}}=1 \mu \mathrm{m}\right)$. La première détermination $-2,0 \mathrm{pm} / \mathrm{V}$ est en accord avec les valeurs rapportées dans la littérature pour des films obtenus par pulvérisation DC réactive pulsée à $200{ }^{\circ} \mathrm{C}$ et $90{ }^{\circ} \mathrm{C}, d_{31}=-2,0 \pm 0,1 \mathrm{pm} . \mathrm{V}^{-1}$ et $d_{31}=-1,6 \pm 0,1 \mathrm{pm} . \mathrm{V}^{-1}$ respectivement [50] et pour des films élaborés par croissance épitaxique, $d_{31}=$ $-1,8 \pm 0,1 \mathrm{pm} . \mathrm{V}^{-1}$ [51]. Cependant, dans cette étude tous les films sont déposés sur un substrat relativement épais ce qui inhibe la déformation dans le plan du film et réduit donc la déformation selon l'axe $\vec{c}$ perpendiculaire au plan du film. Ainsi, la valeur du coefficient mesuré sur ces films $d_{31}^{\mathrm{c}}$ est plus faible que celle d'un matériau volumique $d_{33}^{\mathrm{b}}$ libre de toutes conditions aux limites. De plus, pour un cristal de type Wurzite $d_{31}=-\frac{1}{2} d_{33}$. Il est alors possible de calculer $d_{33}^{\mathrm{b}}$ à partir de $d_{13}^{\mathrm{c}}$ mesuré sur les films avec la relation $[50,51]$ :

$$
d_{33}^{\mathrm{b}}=-2 d_{13}^{\mathrm{c}}\left(\frac{S_{11}^{\mathrm{E}}+S_{12}^{\mathrm{E}}}{S_{11}^{\mathrm{E}}+S_{12}^{\mathrm{E}}+\alpha S_{13}^{\mathrm{E}}}\right)
$$

$\alpha$ est un facteur dépendant des conditions de liaison du film au substrat $(0<\alpha<1)$ et les $S_{i j}$ sont les compliances élastiques à champ électrique constant déduites des valeurs des $C_{i j}$ (Tab. 1, $[35,36]$ ). L'application numérique de l'équation (14) pour un film parfaitement encastré, $\alpha=1$, donne $d_{33}^{\mathrm{b}}=5,7 \mathrm{pm} . \mathrm{V}^{-1}$ pour $d_{31}^{\mathrm{c}}=-2,0 \mathrm{pm} . \mathrm{V}^{-1}$ et $d_{33}^{\mathrm{b}}=3,7 \mathrm{pm} \cdot \mathrm{V}^{-1}$ pour $d_{13}^{\mathrm{c}}=-1,3 \mathrm{pm} \cdot \mathrm{V}^{-1}$. La première valeur est très proche de celle déterminée pour un matériau volumique : $d_{33}^{\mathrm{b}}=5,6 \mathrm{pm} . \mathrm{V}^{-1}$ [51] et $d_{33}^{\mathrm{b}}=5,0 \mathrm{pm} \cdot \mathrm{V}^{-1}[52]$.

Cependant, pour le wafer 1 , la valeur de $d_{33}^{\mathrm{b}}$ est un peu faible comparée à celle d'un matériau volumique, ce qui tendrait à montrer que le procédé de déposition nécessiterait d'être encore optimisé. Une autre explication possible pourrait être la dépendance du coefficient $\alpha$ avec l'épaisseur du film. Le facteur $\alpha$ est plus grand pour une plus faible épaisseur, ainsi pour la même valeur de $d_{33}^{\mathrm{b}}, d_{31}^{\mathrm{c}}$ devrait décroître avec $\alpha$ puisque $S_{13}$ est négatif. 


$$
\begin{aligned}
\Delta T & =\frac{E_{\mathrm{eq}} h_{\mathrm{eq}}^{3}\left(E_{\mathrm{f}} h_{\mathrm{f}}+E_{\mathrm{s}} h_{\mathrm{s}}+E_{\mathrm{e}} h_{\mathrm{e}}\right)}{3\left[E_{\mathrm{s}} h_{\mathrm{s}}^{2}-\left(E_{\mathrm{f}} h_{\mathrm{f}}+E_{\mathrm{e}} h_{\mathrm{e}}\right)\left(h_{\mathrm{e}}+h_{\mathrm{f}}\right)\right]\left[\alpha_{\mathrm{s}}\left(E_{\mathrm{f}} h_{\mathrm{f}}+E_{\mathrm{e}} h_{\mathrm{e}}\right)-\left(E_{\mathrm{f}} \alpha_{\mathrm{f}} h_{\mathrm{f}}+E_{\mathrm{e}} \alpha_{\mathrm{e}} h_{\mathrm{e}}\right)\right] L^{2}} \\
A_{\exp } U^{2} & =A_{\mathrm{exp}}^{*} U^{2}
\end{aligned}
$$
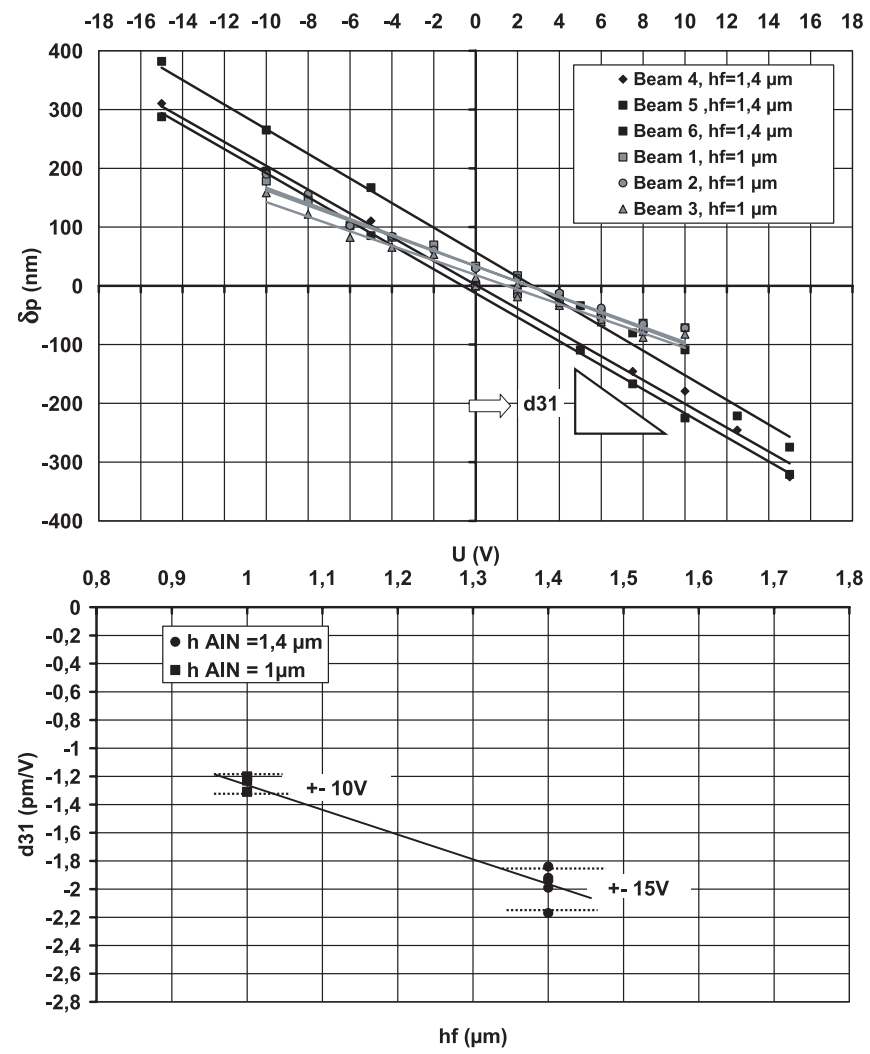

Fig. 11. Déplacement induit par l'application d'une tension continue sur des poutres de $800 \mu \mathrm{m}$ de long pour des épaisseurs d'AlN de $1 \mu \mathrm{m}$ et de 1,4 $\mu \mathrm{m}$ (a) coefficient piézoélectrique $d_{31}$ fonction de l'épaisseur des films (b).

Ceci pourrait conduire à une relation entre la valeur des coefficients piézoélectriques et l'épaisseur du film.

\subsection{Effet Joule sur quelques poutres}

La résistance $R$ de chacun des réseaux de poutres des wafers 1 et 2 a été mesurée. Généralement, $R=$ $\infty$ puisque les électrodes ne se touchent pas et que le film d'AlN agit comme isolant. Cependant, sur quelques réseaux, $R$ est fini, ce qui montre que les électrodes sont en court-circuit. Dans ce cas, lorsqu'une tension est appliquée, il apparaît un déplacement important $\delta>>\delta$ p proportionnel au carré de la tension appliquée (Fig. 12a) et qui a l'effet Joule pour origine. On remarque également qu'une tension de $15 \mathrm{~V}$ est quelquefois trop élevée pour les poutres avec $h_{\mathrm{f}}=1 \mu \mathrm{m}$, conduisant à un endommagement des électrodes et peut-être du film d'AlN. Ainsi, on passe d'un comportement linéaire à un comportement parabolique.

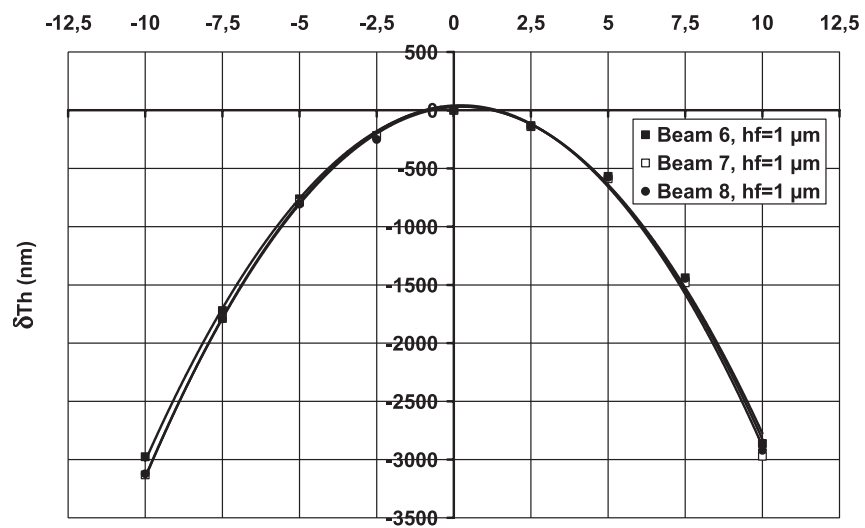

$\mathrm{u}(\mathrm{V})$

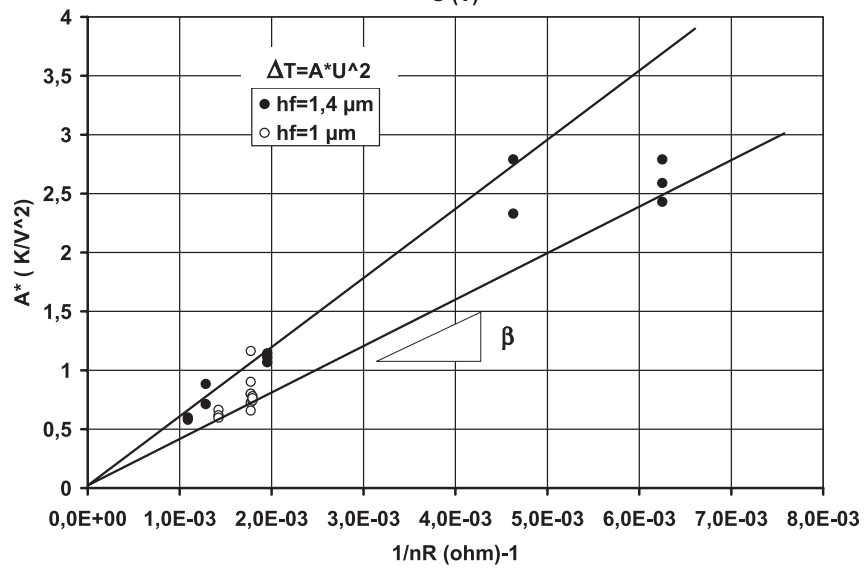

Fig. 12. Déplacement induit par l'application d'une tension sur les réseaux de poutres en court-circuit (a). Évolution du coefficient $A_{\exp }^{*}$ en fonction de l'inverse de la résistance électrique mesurée aux bornes des réseaux court-circuités (b).

De la figure $12 \mathrm{a}$ on peut déduire la relation :

$$
\delta_{\mathrm{Th}}=A_{\mathrm{exp}} U^{2}
$$

qui combinée avec l'équation (5) donne :

$$
\text { voir équation (16) ci-dessus. }
$$

Un modèle thermique simpliste; un mur chauffé sur l'un de ses côtés par une résistance électrique $r$ montre que : $\Delta T=\beta \frac{U^{2}}{r}$ et donc, puisque les résistances des $n$ poutres formant un réseau sont associées en parallèle :

$$
A_{\text {exp }}^{*}=\frac{\beta}{n R}
$$

La représentation $A_{\text {exp }}^{*}=f(1 / n R)$ pour tous les réseaux de poutres en court-circuit, généralement formé de $n=8$ à 9 poutres, est donnée sur la figure $12 \mathrm{~b}$. La pente des 
droites tracées donne $400<\beta<590 \mathrm{~K} . \mathrm{W}^{-1}$. Ce modèle simpliste conduit à :

$$
\beta=\frac{h_{\mathrm{Si}}}{\lambda_{\mathrm{Si}} b L_{\mathrm{s}}}
$$

où $\lambda_{\mathrm{Si}}$ est la conductibilité thermique du silicium, $b$ la largeur des poutres et $L_{\mathrm{S}}$ la longueur à partir de l'encastrement où s'est formé le court-circuit. En prenant $\lambda_{\mathrm{Si}} \cong 150 \mathrm{~W} / \mathrm{m} \mathrm{K}\left(T \cong 50{ }^{\circ} \mathrm{C}\right)$ on trouve que $3 \mu \mathrm{m}$ $<L_{\mathrm{S}}<6 \mu \mathrm{m}$, ce qui est très proche de l'encastrement. Ceci se vérifie sur les images MEB des poutres qui sont passées d'un comportement linéaire à un comportement parabolique après endommagement et qui présentent des zones d'endommagement bien visibles de type oxydation liées à l'échauffement au voisinage de l'encastrement des poutres.

\section{Conclusion}

On présente le procédé de fabrication de micropoutres multimorphes d'AlN pouvant être activées grâce aux propriétés piézoélectriques du film. Le CrNi comme électrode inférieure semble être un bon choix puisque dans ce cas l'AlN présente une orientation très bien définie de type (002), une valeur peu dispersée et élevée de module d'Young correspondant à cette orientation et enfin des contraintes résiduelles plus faibles qu'avec le Pt. L'influence de l'épaisseur du film d'AlN sur l'amplitude de ces contraintes a aussi été étudiée et dès lors il semble possible de réaliser en optimisant l'épaisseur des films d'AlN et des électrodes en $\mathrm{CrNi}$ des structures avec des contraintes globales très faibles. Le coefficient de dilatation des films a également été déterminé grâce à des mesures à différentes températures. Les dispositifs réalisés avec la plus forte épaisseur d'AlN ( $\left.h_{\mathrm{f}}=1,4 \mu \mathrm{m}\right)$ présentent les meilleures propriétés piézoélectriques avec un coefficient $d_{31}=-2,0 \mathrm{pm} \cdot \mathrm{V}^{-1}$, ce qui conduit à un $d_{33}^{\mathrm{b}}$ volumique très proche de celui des céramiques AlN massives. À noter que tous les paramètres ont été calculés avec des équations analytiques non simplifiées tenant compte du substrat, du film et des électrodes. D'un point de vue matériau, pour cette orientation (002), les films d'AlN présentent des propriétés électro-thermo-mécaniques, $C_{i j}$, $\alpha, d_{31}^{\mathrm{b}}$ voisines de celles du matériau volumique.

\section{Références}

[1] M. Clement, L. Vergara, J. Sangrador, E. Iborra, A. SanzHervas, SAW characteristics of AlN films sputtered on silicon substrates, Ultrasonics 42 (2004) 403-407

[2] T. Palacios, F. Calle, E. Monroy, J. Grajal, M. Eickhoff, O. Ambacher, C. Prieto, Nanotechnology for SAW devices on AIN epilayers, Mat. Sci. and Eng. B 93 (2002) $154-158$

[3] Ju-Hyung Kim, Si-Hyung Lee, Jin-Ho Ahn, Jeon-Kook Lee, AlN piezoelectric materials for wireless communication thin film components, J. Ceram. Processing Research 3 (2002) 25-28
[4] K.M. Lakin, J. Belsick, J.F. McDonald, K.T. McCarron, Improved bulk wave resonator coupling coefficient for wide bandwidth filters, IEEE Ultrasonics Symp. 9 (2001) $3 \mathrm{E}-5$

[5] A. Choujaa, N. Tirole, C. Bonjour, G. Martin, D. Hauden, P. Blint, A. Cachard, C. Pommier, AlN/silicon Lamb-wave microsensors for pressure and gravimetry measurements, Sens. and Actuators A 46-47 (1995) $179-182$

[6] T. Laurent, F.O. Bastien, J.-C. Pommier, A. Cachard, D. Remiens, E. Cattan, Lamb wave and plate mode in $\mathrm{ZnO} /$ silicon and AIN/silicon membrane, Application to sensors able to operate in contact with liquid, Sens. and Actuators A 87 (2000) 26-37

[7] D. Ruffieux, M.A. Dubois, N.F. de Rooij, An AIN piezoelectric microactuator array, IEEE MicroElectro Mech. Syst., Miyazaki, Japan, Jan. 23-27 (2000) 662-667

[8] M.A. Dubois, P. Muralt, Properties of AlN thin films for piezoelectric transducers and microwave filter applications, Appl. Phys. Lett. 74 (1999) 3032-3034

[9] S. Bargiel, D. Heinis, C. Gorecki, A. Gôrecka-Drzazga, J.A. Dziuban, M. Jôzwik, A micromachined silicon-based probe for a scanning near-field optical microscope onchip, Measurement Sci. Techn. 17 (2006) 32-37

[10] M. Ishihara, S.J. Li, H. Yumoto, K. Akashi, Y. Ide, Control of preferential orientation of AlN films prepared by the reactive sputtering method, Thin Solid Films 316 (1998) 152-157

[11] X.H. Xu, H.S. Wu, C.J. Zhang, Z.H. Jin, Morphological properties of AlN piezoelectric thin films deposited by DC reactive magnetron sputtering, Thin Solid Films 388 (2001) 62-67

[12] M. Akiyama, C.N. Xu, K. Nonaka, K. Shoku, T. Watonabe, Statistical approach for optimizing sputtering conditions of highly oriented aluminium nitride thin films, Thin Solid Films 315 (1998) 62-65

[13] K. Kusaka, D. Taniguchi, T. Hanakusa, K. Tomiraga, Effect of input power on crystal orientation an residual stress in AlN film deposited by DC sputtering, Vacuum 59 (2000) 806-813

[14] M.A. Auger, L. Vazquez, M. Jergel, O. Sanchez, J.M. Albella, Structure and morphology evolution of AlN films grown by DC sputtering, Surf. Coat. Techn. 180-181 (2004) 140-144

[15] V.M. Pantojas, W. Otano-Rivera, J.W. Caraballe, Statistical analysis of the effect of deposition parameters on the preferred orientation of sputtered AlN films, Thin Solid Films 492 (2005) 118-123

[16] C. Gorecki, M. Jôzwik, L. Salbut, Multifunctional interferometric platform for on-chip testing the micromechanical properties of MEMS/MOEMS, J. Microlithography, Microfabrication, and Microsystems 4 (2005), 1-10

[17] L. Salbut, K. Patorski, M. Jôzwik, J. Kacperski, C. Gorecki, A. Jacobelli, T. Dean, Active microelement testing by interferometry using time-average and quasi-stroboscopic techniques, SPIE Microsystems Eng.: Metrology and Inspection III 5145 (2003) 23-32

[18] W.C. Oliver, G.M. Pharr, An improved technique for determining hardness and elastic modulus using load and displacement sensing indentation experiments, J. Mater. Res. 1 (1992) 1564-1583 
[19] M.S. Bobji, S.K. Biswas, Hardness of a surface containing uniformly spaced pyramidal asperities, Trib. Lett. 7 (1999) 51-56

[20] M. Qasmi, P. Delobelle, Influence of the average roughness $R_{\mathrm{ms}}$ on the precision of the Young's modulus and hardness determination using nano-indentation technique with a Berkovich indenter, Surf. Coat. Techn. 201 (2006) 1191-1199

[21] G.G. Stoney, The tension of metallic films deposited by electrolysis, Proc. R. Soc. London Serie A 82 (1909) 172

[22] K. Röll, Analysis of stress and strain distribution in thin films and substrates, J. Appl. Phys. 47 (7) (1976) 3224-3229

[23] P.H. Townsend, D.M. Barnett, T.A. Brunner, Elastic relationships in layered composite media with approximation for the case of thin films on a thick substrate, J. Appl. Phys. 62 (1987) 4438-4444

[24] J.H. Jou, L. Hsu, Stress analysis of elastically anisotropic bilayer structures, J. Appl. Phys. 69 (1991) 1384-1388

[25] J.G. Smits, W.S. Choi, The constituent equations of piezoelectric heterogeneous bimorphs, IEEE Trans. Ultrasonics, Ferroelectrics and Frequency Control 38 (1991) 256-270

[26] Q.M. Wang, L.E. Cross, Constitutive equations of symmetrical triple layer piezoelectric benders, IEEE Trans. Ultrasonics, Ferroelectrics and Frequency Control 46 (1999) 1343-1351

[27] K. Yao, W. Zhu, K. Uchino, Z. Zhang, L.C. Lim, Design and fabrication of a high performance multilayer piezoelectric actuator with bending deformation, IEEE Trans. Ultrasonics, Ferroelectrics and Frequency Control 46 (1999) 1020-1027

[28] S.H. Chang, C.C. Chou, Electromechanical analysis of an asymmetric piezoelectric elastic laminate structure: Theory and experiment, IEEE Trans. Ultrasonics, Ferroelectrics and Frequency Control 46 (1999) 441-451

[29] V. Walter, Thesis Univ. Franche-Comté, Caractérisation et modélisation électromécanique de dépots de couches épaisses sérigraphiées sur substrat d'alumine, Application au contrôle de forme et à l'amortissement actif d'un bimorphe, 2001

[30] V. Walter, P. Delobelle, P. Le Moal, E. Joseph, E. Collet, A piezo-mechanical characterization of PZT thick films screen-printed on alumina substrate, Sens. and Actuators A 96 (2002) 157-166

[31] J.J. Vlassak, W.D. Nix, Measuring the elastic properties of anisotropic materials by means of indentation experiments, J. Mech. Phys. Sol. 42 (1994) 1223-1245

[32] J.J. Vlassak, W.D. Nix, Indentation modulus of elastically anisotropic half-spaces, Phil. Magn. A 67 (1993) 1045-1056

[33] A. Delafargue, F.J. Ulm, Explicit approximations of the indentation modulus of elastically orthotropic solids for conical indenters, Int. J. Solids Struct. 41 (2004) 7351-7360

[34] P. Delobelle, E. Fribourg-Blanc, D. Remiens, Mechanical properties determined by nanoindentation tests of PZT and PMNT sputtered thin films, Thin Solid Films 515 (2006) 1385-1393

[35] G. Carlotti, F.S. Hickernell, H.M. Liaw, L. Palmieri, G. Socino, E. Verona, The elastic constants of sputtered aluminium nitride films, IEEE Ultrasonics Symp. 1 (1995) 353-356

[36] K. Tsubouchi, K. Sugai, N. Mikoshida, AIN material constants evaluation and SAW properties on $\mathrm{AlN} / \mathrm{Al}_{2} 0_{3}$ and AlN/Si, IEEE Ultrasonics Symp. 1 (1981) 375-380

[37] A.F. Wright, Elastic properties of zinc-blende and wurtzite AIN, GaN and InN, J. Appl. Phys. 82 (1997) 2833-2839

[38] L. Robert, P. Delobelle, Internal report, unpublished work, 2001

[39] R.B. King, Elastic analysis of some punch problems for layered medium, Int. J. Solids Struct. 23 (1987) $1657-1664$

[40] R. Saha, W.D. Nix, Effects of the substrate on the determination of thin film mechanical properties by nanoindentation, Acta Mater. 50 (2002) 23-28

[41] Z. Xue, Y. Huang, K.C. Hwang, M. Li, The influence of indenter tip radius on the micro-indentation hardness, J. Eng. Mat. Techn. 124 (2002) 371-379

[42] Q.M. Wang, Y. Ding, Q. Chen, M. Zhao, J. Cheng, Crystalline orientation dependence of nanomechanical properties of $\mathrm{Pb}(\mathrm{Zr} \mathrm{Ti}) 0_{3}$ thin films, Appl. Phys. Lett. 86 (2005) 162903

[43] P. Delobelle, G.S. Wang, E. Fribourg-Blanc, D. Remiens, Indentation modulus and hardness of $\mathrm{Pb}(\mathrm{Zr} \mathrm{Ti}) \mathrm{O}_{3}$ solgel films deposited on $\mathrm{Pt}$ and $\mathrm{LuNiO}_{3}$ electrodes. An estimation of the $C_{i j}$ compliances, J. Europ. Ceram. Soc. 27 (2007) 223-230

[44] W. Xiao, X. Jiang, Optical and mechanical properties of nanocrystalline aluminium oxynitride films prepared by electron cyclotron resonance plasma enhanced chemical vapor deposition, J. Crystal Growth 264 (2004) 165-171

[45] S. Dagdag, J. Alexis, J.D. Beguin, J.A. Petit, M. Ferrato, J. Durand, Propriétés mécaniques de revêtements d'AlN sur carbure de silicium fritté par dépôt chimique en phase vapeur assisté plasma, Matériaux 2006, 13-17 Nov. 2006, Dijon, France

[46] W.J. Meng, J.A. Sell, G.L. Eesley, T.A. Perry, Measurement of intrinsic stresses during growth of aluminium nitride thin films by reactive sputter deposition, J. Appl. Phys. 74 (1993) 2411-2414

[47] A. Andrei, K. Krupa, M. Jozwik, L. Nieradko, C. Gorecki, L. Hirsinger, P. Delobelle, Fabrication, characterization and reliability study of AlN driven cantilevers, Proc. of SPIE, vol. 6188 April 2006, Strasbourg

[48] www.ceramic_center.com/cttc_pdf/nitrure_al.pdf

[49] MEMS and Nanotechnology Clearinghouse, www.memsnet.org/material/aluminiumnitride, MEMS Exchange (2001)

[50] L.L. Guy, S. Muensit, E.M. Goldys, Extensional piezoelectric coefficients of gallium nitride and aluminium nitride, Appl. Phys. Lett. 75 (1999) 4133-4135

[51] C.M. Lueng, H.L.W. Chan, S. Surya, C.L. Choi, Piezoelectric coefficient of aluminium nitride and gallium nitride, J. Appl. Phys. 88 (2000) 5360-5363

[52] C.J. Ho, T.K. Shing, P.C. Li, Preferred orientation control and characterization of AlN thin films using reactive sputtering, J. Mat. Sci. and Eng. 7 (2004) 1-4 